University of Texas at El Paso

ScholarWorks@UTEP

\title{
$5-2019$
}

\section{How Earthquake Risk Depends on the Closeness to a Fault: Symmetry-Based Geometric Analysis}

\author{
Aaron A. Velasco \\ The University of Texas at El Paso, aavelasco@utep.edu \\ Solymar Ayala Cortez \\ The University of Texas at El Paso, sayalacortez@miners.utep.edu \\ Olga Kosheleva \\ The University of Texas at El Paso, olgak@utep.edu \\ Vladik Kreinovich \\ The University of Texas at El Paso, vladik@utep.edu
}

Follow this and additional works at: https://scholarworks.utep.edu/cs_techrep

Part of the Computer Sciences Commons

Comments:

Technical Report: UTEP-CS-19-25a

To appear in: Michael Beer and Enrico Zio (eds.) Proceedings of the 29th European Safety and

Reliability Conference ESREL'2019, Hannover, Germany, September 22-26, 2019

\section{Recommended Citation}

Velasco, Aaron A.; Cortez, Solymar Ayala; Kosheleva, Olga; and Kreinovich, Vladik, "How Earthquake Risk Depends on the Closeness to a Fault: Symmetry-Based Geometric Analysis" (2019). Departmental Technical Reports (CS). 1313.

https://scholarworks.utep.edu/cs_techrep/1313

This Article is brought to you for free and open access by the Computer Science at ScholarWorks@UTEP. It has been accepted for inclusion in Departmental Technical Reports (CS) by an authorized administrator of ScholarWorks@UTEP. For more information, please contact Iweber@utep.edu. 


\title{
How Earthquake Risk Depends on the Closeness to a Fault: Symmetry-Based Geometric Analysis
}

\author{
Aaron Velasco and Solymar Ayala Cortez \\ Department of Geological Sciences, University of Texas at El Paso, USA. \\ E-mail:aavelasco@utep.edu, sayalacortez@miners.utep.edu \\ Olga Kosheleva \\ Department of Teacher Education, University of Texas at El Paso, USA. E-mail: olgak@utep.edu \\ Vladik Kreinovich \\ Department of Computer Science, University of Texas at El Paso, USA. E-mail: vladik@utep.edu
}

Earthquakes can lead to a huge damage - and the big problem is that they are very difficult to predict. To be more precise, it is very difficult to predict the time of a future earthquake. However, we can estimate which earthquake locations are probable. In general, earthquakes are mostly concentrated around the corresponding faults. For some faults, all the earthquakes occur in a narrow vicinity of the fault, while for other faults, areas more distant from the fault are risky as well. To properly estimate the earthquake's risk, it is important to understand when this risk is limited to a narrow vicinity of a fault and when it not thus limited.

This problem has been thoroughly studied for the most well-studied fault in the world: San Andreas fault. This fault consists of somewhat different Northern and Southern parts. The Northern part is close to a straight line, and in this part, earthquake are mostly limited to a narrow vicinity of this line. In contrast, the Southern part is different: it is curved, and earthquakes can happen much further from the main fault. In this paper, we provide a general general explanation for this phenomenon. The existence of such a general explanations makes us expect that the same phenomenon will be observed at other not-so-well-studied faults as well.

Keywords: Earthquake risk, geometric approach, geometric approach

\section{Formulation of the Practical Problem}

\subsection{Earthquake prediction is an important problem}

Earthquakes can lead to a huge damage - and the big problem is that they are very difficult to predict. To be more precise, it is very difficult to predict the time of a future earthquake. However, we can estimate which earthquake locations are probable; see, e.g., Rasulo et al. (2015, 2016).

\subsection{Specific aspect of earthquake prediction: localization}

In general, earthquakes are mostly concentrated around the corresponding faults. However:

- for some faults, all the earthquakes occur in a narrow vicinity of the fault, while

- for others, areas more distant from the fault are risky as well.

To properly estimate the earthquake's risk, it is important to understand when this risk is limited to a narrow vicinity of a fault and when this risk is not thus limited.

\subsection{Case study: San Andreas fault}

This problem has been thoroughly studied for the most well-studied fault in the world: San Andreas fault; see, e.g., Wallace (1990); Lynch (2015); Schulz and Wallace (2016). This fault consists of somewhat different Northern and Southern parts:

- The Northern part is close to a straight line. In this part, the fault itself is narrow - e.g., it is less than a mile wide in the Olema Trough part, and earthquakes are mostly limited to a narrow vicinity of this line, within \pm 10 miles.

- The Southern part is geometrically different: it is curved. In the South, the fault itself is much wider - e.g., it is many miles across in the Salton Trough part, and earthquakes can happen much further from the main fault, at a distance up to 30 miles away.

Proceedings of the 29th European Safety and Reliability Conference.

Edited by Michael Beer and Enrico Zio

Copyright (C) 2019 by ESREL2019 Organizers. Published by Research Publishing, Singapore

ISBN: 981-973-0000-00-0 :: doi: 10.3850/981-973-0000-00-0_tr19-25a 


\subsection{Resulting problem}

It would be great to find a general explanation for this phenomenon, so that we would be able to better understand other, not so well-studied faults.

\subsection{What we do in this paper}

In this paper, we show that the above phenomenon has a general geometric explanation and can, thus, probably be extended to other faults as well.

\section{Main Idea: Using Symmetries}

\subsection{What we will do}

In this research, we will be using the idea of symmetries.

Symmetries is one of the fundamental - and one of the most successful - ideas in physics in general; see, e.g., Feynman et al. (2005); Thorne and Blandford (2017). However, since the idea of symmetries is not yet as popular - and even not yet well known - in engineering and geosciences, we need to explain this idea in some detail.

\subsection{How do we make predictions in the first place?}

The idea of symmetry comes from the way we make predictions. For example, if you have a pen in your hand and you drop it, it will fall down with the acceleration of $9.81 \mathrm{~m} / \mathrm{sec}^{2}$. If you rotate yourself by 90 degrees and repeat the same experiment, you will get the same result. You can rotate yourself by other angles - and still get the same results. So, after several such experiments, you can reasonably confidently conclude that the pen-falling-down process does not change if we simply rotate the whole setting by any angle.

Similarly, if you step a few steps forward, backward, to the right, or to the left, and repeat the same pen-falling-down experiment, you will get the exact same result. If you repeat this experiment in Hannover, Germany, instead of El Paso, Texas, the result will be the same (let us ignore for now the minor difference in the gravitational fields - it is minor for the purpose of this experiment but it provides very important geophysical information).

Thus, we can conclude that the results of the experiment do not change if we shift the experiment to a different location.

This is how we, in general, make predictions. We observe that some phenomenon does not change if we perform some changes ("transformations") to its setting. Then, we can conclude that in the future, if we perform a similar transformation, we should get the same result.

The experiments do not have to be as simple as dropping a pen. For example, how do we know that Ohm's law - according to which the voltage
$V$ is proportional to the current $I-$ is valid? Ohm observed it in Denmark, then different researchers observed the exact same phenomenon in different locations - so now we can conclude that this law is indeed universally valid.

The symmetries also do not have to be as simple as rotations and shifts. For example, in engineering, many processes do not change if we change the scale - that is why testing a small-size model of a plane helped us to understand how the actual full-size plane will behave in flight.

In physics, there are even more complex examples of symmetries: for example,

- if we replace elementary particles by the corresponding antiparticles, almost all physical processes will remain the same;

- if we invert the flow of time, most equations remains valid, etc.

\subsection{What is symmetry: towards a formal definition}

To describe what is symmetry, we need to have a class of possible transformations - like rotations, shifts, or replacing each particle with its antiparticle.

If two different transformations $T_{1}$ and $T_{2}$ are possible, then we can first perform the first one and then the second one and get a combined transformation $T_{2} T_{1}$ which is called a composition.

We can have a composition of more than two transformations: e.g., if we first apply $T_{1}$, then $T_{2}$, and then $T_{3}$, then we get a composition $T_{3} T_{2} T_{1}$. It is easy to see that we get the same process:

- whether we first apply $T_{2} T_{1}$ and then $T_{3}$, or

- whether we first apply $T_{1}$, and then $T_{3} T_{2}$ :

$$
T_{3}\left(T_{2} T_{1}\right)=\left(T_{2} T_{2}\right) T_{1} .
$$

In mathematical terms, this means that the composition operation is associative.

Also, most transformation are reversible:

- if we rotate by 90 degrees to the right, we can then rotate by 90 degrees to the left and thus come back to the original position;

- if we go forward 10 meters, we can then go back 10 meters and thus come back to the original position;

- if we replace each particle with its antiparticle, we can then repeat the same replacement and get back the original matter, etc.

This "reversing" transformation - usually denoted by $T^{-1}$ - has the property that it cancels the effect of the original one:

$$
T^{-1} T=T T^{-1}=I,
$$


where I ("identity") indicated a "transformation" that does not change anything. Clearly, for the identity transformation, we have

$$
T I=I T=T
$$

for all $T$.

So, on the class of transformations, we have an associate binary operation for which:

- there is a transformation $I$ with the property

$$
T I=I T=T
$$

for all $T$, and

- for each transformation $T$, there is an "inverse" one $T^{-1}$ for which

$$
T^{-1} T=T T^{-1}=I \text {. }
$$

In mathematics, a pair consisting of a set and a binary operation with these properties is called a group. Thus, possible transformations form a group. This group is usually called a transformation group.

\subsection{How physical laws are described in these terms}

As we have mentioned, many physical laws simply mean that a certain property of the physical world does not change under some class of transformations. In mathematical terms, we can say that that these properties are invariant under the corresponding transformation groups.

In physics, transformations for which some properties are preserves are also called symmetries, and the corresponding transformation group is called a symmetry group.

Comment. These terms are consistent with the usual meaning of the word "symmetry": e.g., when we say that a football is spherically symmetric, we mean that its shape does not change if we rotate it in any way around its center. In this case, rotations are symmetries of this ball.

\subsection{This approach has been very successful in physics}

In the past - starting with Isaac Newton, father of modern physics - new physical theories were usually described in terms of the corresponding differential equations. However, starting from the 1960s quark theory, many physical theories are now formulated exclusively in terms of symmetries - and equations follow from these symmetries.

Moreover, it turned out that many classical physical theories, theories that were originally formulated in terms of differential equations, can be derived from the corresponding symmetries; see, e.g., Finkelstein and Kreinovich (1985); Finkelstein et al. (1986); Kreinovich (1976); Kreinovich and Liu (2017).

\subsection{Symmetries can also explain physical phenomena}

Symmetries can help not only to explain theories, but to explain phenomena as well. For example, there are several dozens theories explaining the spiral structure of many galaxies - including our Galaxy.

It has been shown that all possible galactic shapes - and many other physical properties - can be explained via symmetries; see, e.g., Finkelstein et al. (1997a); Li et al. (2002).

\subsection{Symmetries beyond physics}

Similarly, symmetries can be helpful:

- in biology - where they explain, e.g., Bertalanfi equations describing growth,

- in computer science - when they help with testing programs, and in many other disciplines;

see, e.g., Nguyen and Kreinovich (1997).

\subsection{Symmetries in engineering and data processing}

Symmetries not only explain, they can help design. For example:

- symmetries (including non-geometric ones like scalings) can be used to find an optimal design for a network of radiotelescopes; see, e.g., Kreinovich et al. (2002, 2003);

- symmetries can help to come up with optimal algorithms for processing astroimages; see, e.g., Kosheleva (1998); Kosheleva and Kreinovich (1979).

Natural symmetries can also explain which methods of processing expert knowledge work well and which don't; see, e.g., Kreinovich et al. (1998); Nguyen and Kreinovich (1997, 1998).

\section{Let Us Apply Symmetries to Our Problem}

\subsection{What are the symmetries here?}

Now that we are (hopefully) convinced that symmetries are helpful in many research areas, let us use them in our analysis of the earthquake risk. To apply symmetries, we first need to find out what are the reasonable symmetries here.

To answer this question, let ue recall that, on a very large scale, the Earth's geophysical structure is reasonably homogeneous and isotropic. So, in 
the first approximation, each piece of the Earth surface is symmetric with respect to shifts and rotations.

We also do not have any selected distances, which means that the initial configuration is invariant with respect to scalings, i.e., transformations $x_{i} \rightarrow \lambda \cdot x_{i}$ which correspond to changing the unit of measuring distances.

\subsection{The corresponding symmetry is unstable}

In the ideal case, the Earth would be perfectly symmetric, it will have the exact same properties at each geographic rotation. For example, we would have different layers at exactly the same depth at each location. In particular, we will have the molten material at exactlly the same depth at each location.

However, as geophysicists know, this complete symmetry is unstable. E.g., due to random fluctuations, at some location, magma penetrates higher than in other locations - so in this location, the barrier for magma becomes thinner and thus, easier to penetrate. As a result, the magma from the surrounding areas start flowing into this area and push up even more. So, the initially small perturbation grows and grows - until the magma comes to the Earth's surface as lava from a newborn volcano.

Such increase in asymmetry is ubiquitous in physics, it is known as spontaneous symmetry breaking; see, e.g., Feynman et al. (2005); Thorne and Blandford (2017).

\subsection{Which spontaneous symmetry breakings are most probable}

According to statistical physics (see, e.g., ? and Thorne and Blandford (2017)), the most probable are symmetry breakings that retain the largest number of symmetries.

This may sound like a very abstract and not very intuitive idea, but many examples of it are very intuitive. For example, in the states of matter:

- At low (or sometimes at very low) temperatures, every material becomes a crystal, i.e., has many symmetries.

- In the liquid state, there are fewer symmetries: e.g., volume is preserved but not much else.

- Finally, in the state of gas, there are, in effect, no symmetries at all.

And indeed, the transition from one state to another follows the above general idea: when heated, the solid body usually turns first into liquid (i.e., state with some symmetries remaining) and not directly into gas (state with no symmetries).

Let us apply this principle to our situation as well.

\subsection{What are the resulting shapes}

We start with a state which is invariant under arbitrary shifts, rotations, and scalings. After the spontaneous symmetry breaking, according to the above physical principle, the most probable state will still have some symmetries.

Let us denote the corresponding symmetry group by $G$. This remaining symmetry means that if we have a perturbation at some location $a$, then, for each transformation $g \in G$, we will have a similar perturbation at the location $g(a)$ obtained from $a$ by applying the transformation $g$. Thus, together with each location $a$, the set of all locations where we observe a similar perturbation contains the whole set

$$
S=G(a) \stackrel{\text { def }}{=}\{g(a): a \in G\} .
$$

In mathematical terms, this set is called an orbit - namely, it is an orbit containing the location $a$. Thus, we can conclude that the resulting shape consists of orbits of the remaining symmetry group $G$.

\subsection{Orbits are themselves invariant}

It is important to mention that each orbit $S$ is itself invariant with respect to the symmetry group $G$. In precise terms, for each transformation $g_{0} \in G$, we have

$$
S=g_{0}(S) .
$$

This fact is easy to prove. In general, to prove that two sets are equal, we need to prove that:

- every element from the first set is included in the second one, and

- vice versa, every element of the second set is included in the first set.

Let us show that this is the case here.

- If $b \in S$, this means that $b=g(a)$ for some transformation $g \in G$. Then, for $g^{\prime} \stackrel{\text { def }}{=} g_{0}^{-1} g \in G$, we have

$$
\begin{aligned}
& c \stackrel{\text { def }}{=} g^{\prime}(a)=g^{-1}(g(a)) \in S=G(a) \\
& \quad \text { and } \\
& g_{0}(c)=g_{0}\left(g_{0}^{-1}(g(a))=g(a)=b .\right.
\end{aligned}
$$

Thus, the element $b$ indeed belongs to the set $g_{0}(S)$.

- Vice versa, let $b \in g_{0}(S)$. This means that $b=g_{0}(c)$ for some $c \in S=G(a)$, and this, in turn, means that $c=g(a)$ for some $g \in G$. Thus, we have

$$
b=g_{0}(c)=g_{0}(g(a))=\left(g_{0} g\right)(a) .
$$


Since $G$ is a group, it contains a composition $g_{0} g$ of its transformations $g_{0}$ and $g$. Thus, indeed, $b=g^{\prime}(a)$ for some $g^{\prime} \in G$ : namely, this is true for $g^{\prime}=g_{0} g$. This implies that $b \in S=G(a)$.

The invariance is proven.

\subsection{So what are the orbits here}

For the group of all shifts, rotations, and scalings on the plane, all subgroups and corresponding orbits are well-known; see, e.g., Finkelstein et al. (1997 a); Li et al. (2002) and references therein. When the group is large enough - e.g., if it contains all shifts - the orbit is the whole plane. The only connected orbits which are different from the whole plane are:

- straight lines,

- half-lines,

- circles, and

- logarithmic spirals - which, in polar coordinates, have the form

$$
\ln (r)=p+q \cdot \varphi
$$

These are indeed typical shapes of a fault: faults are either almost straight lines or curves - shaped like segments of circles or segments of logarithmic spirals.

Comment. Of course, we are only talking about a local shape: a straight line goes all the way to infinity, but a fault is usually a reasonably local phenomenon.

\subsection{Side comment: which fault shape should be more frequent and which is more frequent}

In case of a straight line, we have a 2-D remaining symmetry group:

- a straight line is invariant with respect to shifts along this line, and

- it is also invariant with respect to scalings.

In contrast, circles and logarithmic spirals only have a 1-D symmetry groups:

- a half-line is invariant with respect to scalings,

- a circle is invariant with respect to all rotations around its center, and

- a logarithmic spiral is invariant with respect to combined rotation-and-scaling transformations, namely, a combination of a rotation $\varphi \rightarrow \varphi+\varphi_{0}$ and a scaling

$$
r \rightarrow \exp \left(q \cdot \varphi_{0}\right) \cdot r .
$$

Thus, by using the above-described general physical idea, we can conclude that straight-line faults should be more probable - and thus, more frequent - that the curved-shaped ones.

And indeed, in nature, most faults are close to straight lines, and curves faults are much more rare.

\subsection{What is the shape of the near-fault earthquake activity area}

Let us use the above results to analyze the original problem: what is the shape of the near-fault seimsically active area.

In our analysis of possible fault shapes, we used the statistical-physics idea that the resulting physical system should be invariant with respect to some subgroup of the original symmetry group. We used this idea to derive possible fault shapes, and we concluded that we have four options:

- a straight line (with shifts and scalings),

- a half-line (with scalings only),

- a circle (with rotations), and

- a logarithmic spiral (with combined rotation-and-scaling symmetries).

It is reasonable to conclude that the nearfault earthquake-prone risk region should have the same symmetries as the fault.

- For a straight line, these symmetries are shift and re-scaling, and the only region with the same symmetries is the fault itself - which explains why there is practically no activity outside the fault.

- For half-line, i.e., for a fault with an abrupt end-point - an angular segment has the same symmetries.

- Similarly, for a circle or for a logarithmic spiral, if we start with a different point, we can have another orbit with the same symmetries - e.g., a circular disk has the same symmetries as the circle. Thus, for faults of this shape, earthquakes outside the fault are possible.

\subsection{Conclusion}

For the San Andreas fault, it was observed that:

- for the continuous straight-line fault segment, only a very narrow vicinity of a fault is risk-prone - at distances not exceeding 10 miles from the fault, while

- for the curved-shaped fault segment, earthquakes can also happen at a reasonable distance from the fault, up to 30 miles distance.

In this paper, we show that this empirical phenomenon has a solid geometric explanation - and thus, we expect that the same phenomenon will be observed at other faults as well. 


\section{Acknowledgments}

This work was supported in part by the US National Science Foundation grant HRD-1242122 (Cyber-ShARE Center of Excellence).

The authors are thankful to the anonymous referees for valuable suggestions.

\section{References}

Doser, D., M. A. Khamsi, and V. Kreinovich (1996). Earthquakes and geombinatorics. Geombinatorics 6(2), 48-54.

Feynman, R., R. Leighton, and M. Sands (2005). The Feynman Lectures on Physics, Addison Wesley.

Finkelstein, A., O. Kosheleva, and V. Kreinovich, Astrogeometry: geometry explains shapes of celestial bodies (1997). Geombinatorics VI(4), 125-139.

Finkelstein, A., O. Kosheleva, and V. Kreinovich (1997a). Astrogeometry: towards mathematical foundations. International Journal of Theoretical Physics 36(4), 1009-1020.

Finkelstein, A., and V. Kreinovich. Derivation of Einstein's, Brans-Dicke and other equations from group considerations (1985). In: Y. Choque-Bruhat and T. M. Karade (Eds), On Relativity Theory. Proceedings of the Sir Arthur Eddington Centenary Symposium, Nagpur India 1984, Vol. 2, pp. 138-146, World Scientific.

Finkelstein, A., V. Kreinovich, and R. R. Zapatrin (1986). Fundamental physical equations uniquely determined by their symmetry groups. Springer Lecture Notes in Mathematics, 1214, 159-170.

Kosheleva, O. (1998). Symmetry-group justification of maximum entropy method and generalized maximum entropy methods in image processing, In: G. J. Erickson, J. T. Rychert, and C. R. Smith (Eds.), Maximum Entropy and Bayesian Methods, pp. 101-113, Kluwer.

Kosheleva, O. M., and V. Ya. Kreinovich (1979). A letter on maximum entropy method, Nature 281 (5733), 708-709.

Kreinovich, V. (1976). Derivation of the Schroedinger equations from scale invariance, Theoretical and Mathematical Physics 8(3), 282-285.

Kreinovich, V. and G. Liu (2017). We live in the best of possible worlds: Leibniz's insight helps to derive equations of modern physics, In: R. Pisano, M. Fichant, P. Bussotti, and A. R. E. Oliveira (Eds.), The Dialogue between Sciences, Philosophy and Engineering. New Historical and Epistemological Insights, Homage to Gottfried W. Leibnitz 1646-1716, pp. 207-226, College Publications.

Kreinovich, V., G. C. Mouzouris, and H. T. Nguyen (1998). Fuzzy rule based modeling as a universal approximation tool, In: H. T. Nguyen and M. Sugeno (Eds.), Fuzzy
Systems: Modeling and Control, pp. 135-195, Kluwer.

Kreinovich, V., S. A. Starks, D. Iourinski, O. Kosheleva, and A. Finkelstein (2002). Openended configurations of radio telescopes: towards optimal design. In: Proceedings of the 2002 World Automation Congress WAC'2002, Orlando, Florida, June 9-13, 2002, pp. 101106.

Kreinovich, V., S. A. Starks, D. Iourinski, O. Kosheleva, and A. Finkelstein (2003). Openended configurations of radio telescopes: a geometrical analysis. Geombinatorics 13(2), 7985.

Li, S., Y. Ogura, and V. Kreinovich (2002). Limit Theorems and Applications, Kluwer.

Lynch, D. K. (2015) The Field Guide to the San Andreas Fault, Sunbelt Publications, El Cajon, California.

Nguyen, H. T., and V. Kreinovich (1997). Applications of Continuous Mathematics to Computer Science, Kluwer.

Nguyen, H. T., and V. Kreinovich (1998). Methodology of fuzzy control: an introduction, In: H. T. Nguyen and M. Sugeno (eds.), Fuzzy Systems: Modeling and Control, pp. 19-62, Kluwer.

Rasulo, A., C. Testa, and B. Borzi (2015) Seismic risk analysis at urban scale in Italy. In: Gervasi, O. et al. (eds) Computational Science and Its Applications - ICCSA'2015, Lecture Notes in Computer Science, vol 9157. Springer, Cham, Switzerland.

Rasulo, A., M. A. Fortuna, and B. Borzi (2016) A seismic risk model for Italy. In: Gervasi, O. et al. (eds) Computational Science and Its Applications - ICCSA'2016, Lecture Notes in Computer Science, vol 9788. Springer, Cham, Switzerland.

Schulz, S. S., and R. E. Wallace, The San Andreas Fault, United State Geological Survey, Washington, DC, 2016.

Thorne, K. S., and R. D. Blandford (2017). Modern Classical Physics: Optics, Fluids, Plasmas, Elasticity, Relativity, and Statistical Physics, Princeton University Press.

Wallace, R. I. (1990) The San Andreas Fault System, California, United State Geological Survey, Washington, DC, Publication 1515. 